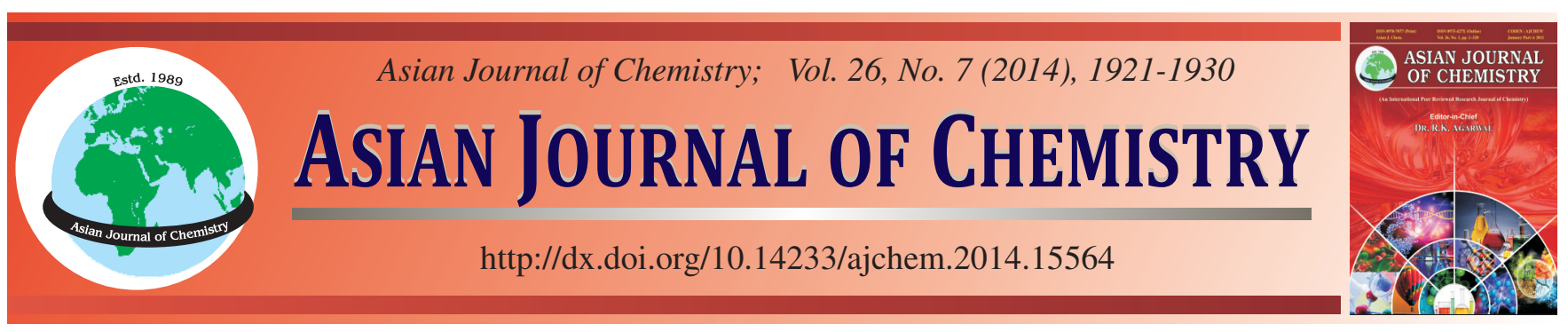

\title{
One-Pot Synthesis of Novel 3,5-Disubstituted-1,2,4-oxadiazoles from Indazole Carboxylic Acid Esters and Amidoximes
}

Udutha Kumara Swamy ${ }^{1, *}$, H. Rama Mohan ${ }^{1}$, U. Viplava Prasad $^{2}$, T. Suresh ${ }^{1}$ and T. Laxmi Kumar ${ }^{1}$

${ }^{1}$ Laurus Labs Private Limited, IKP Knowledge Park, Turkapally, Shameerpet, Hyderabad-500 078, India

${ }^{2}$ Department of Organic Chemistry, Foods, Drugs and Water, Andhra University, Visakhapatnam-530 003, India

*Corresponding author: Fax: +91 40 23480480; Tel: +91 40 30413370; E-mail: kumarudutha@ gmail.com

An efficient and high-yielding one-pot synthesis of 3,5-disubstituted-1,2,4-oxadiazoles from indazole carboxylic acid methyl esters and amidoximes is described. In this study a series of novel 3,5-disubstituted-1,2,4-oxadiazoles (3a-d), (4a-d), (5a-d), (6a-d), (7a-d) were synthesized using amidoximes $\mathbf{2 a - d}$ and indazole carboxylic acid esters (3-6).

Keywords: Amidoximes, Indazole carboxylic acid esters, 1,2,4-Oxadiazoles.

\section{INTRODUCTION}

1,2,4-Oxadiazoles are important class of heterocycles due to their biological importance and are stable to physiological environment. 1,2,4-Oxadiazoles have often been used as bioisosteres of esters and amides ${ }^{1}$ and are found in several drugs and drug leads ${ }^{2}$ which includes potent sphingosine-1phosphate-1 (S1P1) agonist $(\mathbf{I})^{3}$, the metabotropic glutamate subtype 5 (mGlu5) receptor (II) ${ }^{4}$ and muscarinic receptor (III) ${ }^{5}$ for the treatment of Alzheimer's diseases. They can also be found in a number of biologically important molecules, such as serotoninergic $\left(5-\mathrm{HT}_{3}\right)$ antagonists (IV) ${ }^{6}$, benzodiazepine receptor agonists $(\mathbf{V})^{7}$ and dopamine ligands $(\mathbf{V I})^{8}$. Several papers reported the use of 1,2,4-oxadiazole in a number of pharmacologically important molecules, including the design of amino acyl-gly dipeptidomimetics ${ }^{9}$, signal transduction inhibitors ${ }^{10}$, or cell adhesion inhibitors ${ }^{11}$ as antikinetoplastid materials ${ }^{12}, \beta_{3}$ adrenergic receptor ${ }^{13}$, antiinflammatory agents ${ }^{14}$, agrochemical and antifungal activities ${ }^{15-17}$. Compounds containing 1,2,4-oxadiazole moieties are also active against HIV integrase inhibitors ${ }^{18}$ and antituberculostatic agents ${ }^{19}$. They show activity as growth hormone secretagogue ${ }^{20}$ and antitumor agents $^{21}$. They also inhibit SH2 domain of tyrosine kinase ${ }^{22}$, monoamine oxidase $\mathrm{e}^{23}$, human nuetrophil elastase $\mathrm{e}^{24}$, human DNA topoisomerases ${ }^{25}$. As discussed above 1,2,4-oxadiazole moiety shows activities in multiple therapeutic areas, hence an attempt was made at first for the synthesis of novel 3indazolyl-5-aryl-1,2,4-oxadiazoles.<smiles>CC(C)Cc1ccc(-c2nc(-c3ccc(CN4CC(C(=O)O)C4)cc3)no2)cc1</smiles><smiles>N#Cc1cccc(-c2nc(-c3ccccn3)no2)c1</smiles><smiles></smiles><smiles>[R]c1nc(C2=CC(C)c3ccccc32)no1</smiles><smiles>[R]c1nc(-c2ncn3c2CN=C(c2ccccc2)c2ccccc2-3)no1</smiles><smiles>Cc1noc(CC2CC3CCC(C2)N3C)n1</smiles> 


\section{EXPERIMENTAL}

Melting points of newly synthesized compounds were determined in an open glass capillary tubes using Buchi/ Polman melting point apparatus and are uncorrected. Infrared spectra were recorded in potassium bromide pellets with a Perkin IR spectrometer. All ${ }^{1} \mathrm{H}$ NMR and ${ }^{13} \mathrm{C}$ NMR spectra were recorded on a BRUCKER $300 \mathrm{MHz}$ instrument respectively. Chemical shifts are expressed in $\delta$ (ppm) values with tetramethylsilane as an internal standard and the following abbreviations are used: $\mathrm{s}=$ singlet, $\mathrm{d}=$ doublet, $\mathrm{t}=$ triplet, $\mathrm{q}=$ quartet, $\mathrm{m}=$ multiplet, $\mathrm{br}=$ broad, $\mathrm{dd}=$ double doublet and $\mathrm{dt}$ = double triplet. Mass spectra were recorded Agilent-LC-MS instrument giving only $\mathrm{M}^{+}$values using $\left(\mathrm{M}^{+}+1\right)$ mode. TLC was performed with Silica gel GF-254 from Merck \& Co., (Germany). Spots were detected under UV-light or in iodine. The following general experimental procedures followed for the synthesis of all compounds.

General procedure-I (Esterification of acid): To a solution of methyl-nitro benzoic acid $(0.11 \mathrm{~mol})$ in acetone $(200 \mathrm{~mL})$ were added potassium carbonate $(0.22 \mathrm{~mol})$ and was stirred at room temperature for $10 \mathrm{~min}$. Dimethyl sulphate $(0.13 \mathrm{~mol})$ was added to the reaction mixture. The mixture was then stirred for $4 \mathrm{~h}$ at reflux temperature. Reaction was monitored by TLC. After completion, reaction mixture was cooled to room temperature, filtered and washed with acetone. The filtrate was concentrated to get residue and residue was dissolved in ethyl acetate. The organic layer was washed with water three times followed by brine. Organic layer was dried over anhydrous sodium sulfate, filtered and concentrated. The residue was triturated with heptane to give corresponding methyl-methyl nitro benzoate.

Methyl-2-methyl-3-nitro-benzoate (15): Compound 15 was prepared from $\mathbf{1 4}$ following general procedure-I (98\% yield, Cream colour solid, m.p.: 63-65 $\left.{ }^{\circ} \mathrm{C}\right):{ }^{1} \mathrm{H}$ NMR $(300$ $\left.\mathrm{MHz} \mathrm{CDCl}_{3}\right) 7.99$ (dd, 1H, $\left.J=6.69,1.11 \mathrm{~Hz}, \mathrm{Ar}-\mathrm{H}\right), 7.84$ (dd, $1 \mathrm{H}, J=7.02,1.08 \mathrm{~Hz}, \mathrm{Ar}-\mathrm{H}), 7.40$ (t, $1 \mathrm{H}, J=7.02 \mathrm{~Hz}, \mathrm{Ar}-$ $\mathrm{H}), 3.94\left(\mathrm{~s}, 3 \mathrm{H},-\mathrm{OCH}_{3}\right), 2.62$ (s, 3H, - $\left.\mathrm{CH}_{3}\right)$; ESI-MS m/z 196 $(\mathrm{M}+\mathrm{H})^{+}$.

Methyl-3-methyl-4-nitro-benzoate (18): Compound 18 was prepared from $\mathbf{1 7}$ following general procedure-I (92\% yield, Light brown colour solid, m.p.: $\left.79-82{ }^{\circ} \mathrm{C}\right):{ }^{1} \mathrm{H}$ NMR $\left(300 \mathrm{MHz}, \mathrm{CDCl}_{3}\right) 8.03$ (s, 1H, Ar-H), 7.98 (d, 2H, $J=1.26$ $\mathrm{Hz}, \mathrm{Ar}-\mathrm{H}), 3.96$ (s, 3H, $\left.-\mathrm{OCH}_{3}\right), 2.62$ (s, 3H, - $\mathrm{CH}_{3}$ ); ESI-MS $\mathrm{m} / \mathrm{z} 196(\mathrm{M}+\mathrm{H})^{+}$.

Methyl-4-methyl-3-nitro-benzoate (21): Compound 21 was prepared from 20 following general procedure-I (96\% yield, Light brown colour solid, m.p.: 48-52 $\left.{ }^{\circ} \mathrm{C}\right):{ }^{1} \mathrm{H}$ NMR $(300 \mathrm{MHz}$, $\left.\mathrm{CDCl}_{3}\right) 8.60(\mathrm{~d}, 1 \mathrm{H}, J=1.68 \mathrm{~Hz}, \mathrm{Ar}-\mathrm{H}), 8.13(\mathrm{dd}, 1 \mathrm{H}, J=6.24$, $1.77 \mathrm{~Hz}, \mathrm{Ar}-\mathrm{H}), 7.43$ (d, 1H, J = $7.98 \mathrm{~Hz}, \mathrm{Ar}-\mathrm{H}), 3.95$ (s, 3H, $\left.-\mathrm{OCH}_{3}\right), 2.65\left(\mathrm{~s}, 3 \mathrm{H},-\mathrm{CH}_{3}\right)$; ESI-MS m/z $196(\mathrm{M}+\mathrm{H})^{+}$.

Methyl-3-methyl-2-nitro-benzoate (24): Compound 24 was prepared from $\mathbf{2 3}$ following general procedure-I (90\% yield, Light brown colour solid, m.p.: 71-74 $\left.{ }^{\circ} \mathrm{C}\right):{ }^{1} \mathrm{H}$ NMR (300 MHz, $\left.\mathrm{CDCl}_{3}\right)$ 7.86-7.83 (m, 1H, Ar-H), 7.51-7.42 (m, $2 \mathrm{H}, \mathrm{Ar}-\mathrm{H}), 3.89$ (s, 3H, $\left.-\mathrm{OCH}_{3}\right), 2.34$ (s, 3H, - $\left.\mathrm{CH}_{3}\right)$; ESI-MS $m / z 196(\mathrm{M}+\mathrm{H})^{+}$.

General procedure-II (Reduction of nitro group to amine): To a solution of corresponding nitro compound $(0.10$ $\mathrm{mol})$ in ethanol $(200 \mathrm{~mL})$ was added $\mathrm{Fe}(0.40 \mathrm{~mol})$ and heated to reflux. Aqueous $\mathrm{NH}_{4} \mathrm{Cl}(50 \mathrm{~mL}, 1.00 \mathrm{~mol})$ was added slowly drop by drop to the reaction mixture at reflux temperature. After complete addition reaction, mixture was refluxed for $4 \mathrm{~h}$. Reaction was monitored by TLC. After completion, reaction mixture was cooled to room temperature, filtered and washed with ethanol. The filtrate was concentrated to get residue and the residue was dissolved in ethyl acetate. The organic layer was washed with water three times, followed by brine. Organic layer was dried over anhydrous sodium sulfate, filtered and concentrated. The residue was triturated with heptane to give corresponding methyl aminobenzoate.

Methyl-3-amino-2-methyl-benzoate (16): Compound $\mathbf{1 6}$ was prepared from compound $\mathbf{1 5}$ following general procedure-II (86\% yield, brown colour low-melting solid): ${ }^{1} \mathrm{H}$ NMR $\left(300 \mathrm{MHz}, \mathrm{CDCl}_{3}\right) 7.20(\mathrm{dd}, 1 \mathrm{H}, J=6.54,1.23 \mathrm{~Hz}$, Ar-H), 7.05 (t, $1 \mathrm{H}, J=7.86 \mathrm{~Hz}, \mathrm{Ar}-\mathrm{H}), 6.81$ (dd, $1 \mathrm{H}, J=7.23$, $0.63 \mathrm{~Hz}, \mathrm{Ar}-\mathrm{H}), 3.87$ (s, 3H, - $\left.\mathrm{OCH}_{3}\right), 2.33$ (s, 3H, -CH $)$; ESIMS $m / z 166(\mathrm{M}+\mathrm{H})^{+}$.

Methyl-4-amino-3-methyl-benzoate (19): Compound 19 was prepared from compound $\mathbf{1 8}$ following general procedureII (80\% yield, brown color solid, m.p.: $\left.115-120{ }^{\circ} \mathrm{C}\right):{ }^{1} \mathrm{H}$ NMR $\left(300 \mathrm{MHz}, \mathrm{CDCl}_{3}\right) \delta 7.73(\mathrm{~d}, 2 \mathrm{H}, J=9.00 \mathrm{~Hz}, \mathrm{Ar}-\mathrm{H}), 6.62$ (d, $1 \mathrm{H}, J=8.07 \mathrm{~Hz}, \mathrm{Ar}-\mathrm{H}), 4.99$ (bs, 2H, $-\mathrm{NH}_{2}$ ), 3.85 (s, 3H, $\left.-\mathrm{OCH}_{3}\right), 2.17$ (s, 3H, - $\mathrm{CH}_{3}$ ); ESI-MS m/z $166(\mathrm{M}+\mathrm{H})^{+}$.

Methyl-3-amino-4-methyl-benzoate (22): Compound 22 was prepared from compound $\mathbf{2 1}$ following general procedure-II (88\% yield, light brown colour solid, m.p: 115$\left.120{ }^{\circ} \mathrm{C}\right):{ }^{1} \mathrm{H}$ NMR $\left(300 \mathrm{MHz}, \mathrm{CDCl}_{3}\right)$ 7.38-7.33 (m, 2H, ArH), 7.09 (d, 1H, J = 7.71 Hz, Ar-H), 3.87 (s, 3H, $\left.-\mathrm{OCH}_{3}\right), 3.69$ (bs, $2 \mathrm{H},-\mathrm{NH}_{2}$ ), 2.20 (s, 3H, $\left.-\mathrm{CH}_{3}\right)$; ESI-MS m/z $166(\mathrm{M}+\mathrm{H})^{+}$.

Methyl-2-amino-3-methyl-benzoate (25): Compound $\mathbf{2 5}$ was prepared from $\mathbf{2 4}$ following general procedure-II (85 $\%$ yield, Light brown colour low melting solid): ${ }^{1} \mathrm{H}$ NMR (300 $\left.\mathrm{MHz}, \mathrm{CDCl}_{3}\right) \delta$ 7.79-7.76 (m, 1H, Ar-H), 7.20-7.16 (m, 1H, Ar-H), 6.60 (dd, 1H, J = 7.47, $0.45 \mathrm{~Hz}, \mathrm{Ar}-\mathrm{H}), 5.82$ (bs, 2H, $\left.-\mathrm{NH}_{2}\right), 3.8(\mathrm{~s}, 3 \mathrm{H}), 2.16(\mathrm{~s}, 3 \mathrm{H})$; ESI-MS m/z $166(\mathrm{M}+\mathrm{H})^{+}$.

General procedure-III (Synthesis of indazole carboxylic acid methyl ester $(4,5,6))$ : Methyl-amino benzoate $(0.10$ mol) was dissolved in acetic acid $(320 \mathrm{~mL})$ and was cooled to 15 to $20^{\circ} \mathrm{C}$, solution of sodium nitrite $(0.12 \mathrm{~mol})$ in demineralized water $(16 \mathrm{~mL})$ was slowly added over a period of $0.5 \mathrm{~h}$. After complete addition reaction temperature was increased to 25 to $30^{\circ} \mathrm{C}$ and stirring was continued for $2-3 \mathrm{~h}$. The progress of the reaction was monitored by TLC. After completion, reaction mass was filtered and residue washed with acetic acid. Filtrate was concentrated under reduced pressure at below $60{ }^{\circ} \mathrm{C}$ to get residue. To the residue demineralized water was added at room temperature and stirred for $0.5 \mathrm{~h}$. The solid obtained was filtered and washed with water. Crude product was dried under vacuum at $60{ }^{\circ} \mathrm{C}$ for $2 \mathrm{~h}$. Crude product was taken in to $5 \%$ ethyl acetate- $n$-hexane solution and stir for $1 \mathrm{~h}$ at room temperature. Solid was filtered and washed with $5 \%$ ethyl acetate- $n$-hexane mixture. The product was dried under vacuum at below $80{ }^{\circ} \mathrm{C}$ to give corresponding indazoles.

Indazole-4-carboxylic acid methyl ester (4): Compound $\mathbf{4}$ was prepared from compound $\mathbf{1 6}$ following general procedure-III (80\% yield, brown colour solid, m.p.: $\left.150-154{ }^{\circ} \mathrm{C}\right)$ : ${ }^{1} \mathrm{H}$ NMR (300 MHz, $\left.\mathrm{CDCl}_{3}\right) \delta 8.60(\mathrm{~s}, 1 \mathrm{H},-\mathrm{CH}=\mathrm{N}), 7.96$ (dd, 
$1 \mathrm{H}, J=6.66,0.66 \mathrm{~Hz}, \mathrm{Ar}-\mathrm{H}), 7.73$ (d, $1 \mathrm{H}, J=8.40 \mathrm{~Hz}, \mathrm{Ar}-\mathrm{H})$, 7.48 (dd, $1 \mathrm{H}, J=7.32,1.05 \mathrm{~Hz}, \mathrm{Ar}-\mathrm{H}), 4.03$ (s, 3H, $-\mathrm{OCH}_{3}$ ); ESI-MS $m / z, 177(\mathrm{M}+\mathrm{H})^{+}$.

Indazole-5-carboxylic acid methyl ester (5): Compound 5 was prepared from compound $\mathbf{1 9}$ following general procedure-III (85\% yield, brown color solid, m.p.: $\left.>220{ }^{\circ} \mathrm{C}\right):{ }^{1} \mathrm{H}$ NMR (300 MHz, DMSO-d $)_{6} \delta 13.38(\mathrm{~s}, 1 \mathrm{H},-\mathrm{NH}), 8.47(\mathrm{t}$, $1 \mathrm{H}, J=0.78 \mathrm{~Hz},-\mathrm{CH}=\mathrm{N}), 8.23(\mathrm{t}, 1 \mathrm{H}, 1.17 \mathrm{~Hz}, \mathrm{Ar}-\mathrm{H}), 7.89$ (dd, $1 \mathrm{H}, J=7.17,1.62 \mathrm{~Hz}, \mathrm{Ar}-\mathrm{H}), 7.61-7.58$ (m, 1H, Ar-H), $3.84\left(\mathrm{~s}, 3 \mathrm{H},-\mathrm{OCH}_{3}\right)$; ESI-MS m/z $177(\mathrm{M}+\mathrm{H})^{+}$.

Indazole-6-carboxylic acid methyl ester (6): Compound 6 was prepared from compound 22 following general procedure-III (83\% yield, brown color solid, m.p.: 140-144 $\left.{ }^{\circ} \mathrm{C}\right)$ : ${ }^{1} \mathrm{H}$ NMR (300 MHz, DMSO- $d_{6}$ ) $\delta 13.42$ (s, $\left.1 \mathrm{H},-\mathrm{NH}\right), 8.16$ (t, $2 \mathrm{H}, J=10.35 \mathrm{~Hz}, \mathrm{Ar}-\mathrm{H}), 7.86(\mathrm{~d}, 1 \mathrm{H}, J=8.46 \mathrm{~Hz}, \mathrm{Ar}-\mathrm{H}), 7.65$ (dd, $1 \mathrm{H}, J=7.17,1.32 \mathrm{~Hz}, \mathrm{Ar}-\mathrm{H}), 3.86$ (s, 3H, $-\mathrm{OCH}_{3}$ ); ESIMS $m / z 177(\mathrm{M}+\mathrm{H})^{+}$.

General procedure-IV (Synthesis of 1,2,4- oxadiazoles): Method-A: To a mixture of indazole carboxylic acid ester $(2.8 \mathrm{mmol})$ and amidoxime $(2.8 \mathrm{mmol})$ in toluene $(7.5 \mathrm{~mL})$ was added potassium carbonate $(5.6 \mathrm{mmol})$. Reaction mixture was heated to reflux for 6 to $12 \mathrm{~h}$ and reaction progress was monitored by TLC. After completion, reaction mixture was cooled to room temperature and filtered. Filtrate was concentrated to get residue and dissolved in ethyl acetate. Resulting mixture was washed with water and brine. The organic phase dried over anhydrous sodium sulfate. The solvent was removed under reduced pressure and the resulting residue was chromatographed on silica gel, elution with hexane-ethyl acetate $(4: 1)$ to give corresponding 3,5-disubstitued-1,2,4-oxadiazole.

Method-B: To a mixture of indazole carboxylic acid (3.02 $\mathrm{mmol})$ and amidoxime $(3.02 \mathrm{mmol})$ in dichloromethane $(7.5$ $\mathrm{mL})$ was added DCC (3.02 $\mathrm{mmol})$ and HOBt $(3.02 \mathrm{mmol})$. Reaction mixture was stirred at $\mathrm{rt}$ for $4 \mathrm{~h}$ and reaction progress was monitored by TLC. After completion, reaction mixture was filtered. Filtrate was concentrated to get residue and the residue dissolved in pyridine $(2.5 \mathrm{~mL})$. The reaction mixture was heated to reflux for $4 \mathrm{~h}$. Reaction mixture diluted with water and extracted in to ethyl acetate. The organic phase was washed with water and brine. The organic phase dried over anhydrous sodium sulfate. The solvent was removed under reduced pressure and the resulting residue was chromatographed over silica gel, elution with hexane-ethyl acetate (4:1) resulted corresponding 3,5-disubstitued-1,2,4-oxadiazole.

3-Methyl-5-(3-indazolyl)-1,2,4-oxadiazole (3a): Compound $\mathbf{3 a}$ was prepared from compound $\mathbf{3}$ and amidoxime $\mathbf{2 a}$ following general procedure-IV method-A (80\% yield). Cream color solid; IR $\left(\mathrm{KBr}, v_{\max }, \mathrm{cm}^{-1}\right): 3159(\mathrm{NH}), 1605(\mathrm{C}=\mathrm{N}), 1328$, 1058, 744; ${ }^{1} \mathrm{H}$ NMR (300 MHz, DMSO- $d_{6}$ ): $\delta 14.17$ (s, $1 \mathrm{H}$, -NH), 8.19 - 8.15 (m, 1H, Ar-H), 7.72 (d, 1H, J=8.4 Hz, ArH), 7.53-7.48 (m, 1H, Ar-H), 7.39-7.34 (m, 1H, Ar-H), 2.46 $\left(\mathrm{s}, 3 \mathrm{H},-\mathrm{CH}_{3}\right) ;{ }^{13} \mathrm{C}$ NMR $\left(75 \mathrm{MHz}, \mathrm{DMSO}-d_{6}\right): \delta 170.60$, $167.69,141.28,130.25,127.62,123.57,121.54,120.72$, 111.66, 11.62; ESI-MS m/z $201(\mathrm{M}+\mathrm{H})^{+}$.

3-Phenyl-5-(3-indazolyl)-1,2,4-oxadiazole (3b): Compound $\mathbf{3 b}$ was prepared from compound $\mathbf{3}$ and amidoxime $\mathbf{2 b}$ following general procedure-IV method-A (85\% yield). Cream coloured solid; IR (KBr, $\left.v_{\max }, \mathrm{cm}^{-1}\right): 3193(\mathrm{NH}), 1607(\mathrm{C}=\mathrm{N})$, 1351, 1044, 745; ${ }^{1} \mathrm{H}$ NMR (300 MHz, DMSO-d $)$ : $\delta 14.26$ (s,
$1 \mathrm{H},-\mathrm{NH}), 8.33(\mathrm{~d}, 1 \mathrm{H}, J=8.2 \mathrm{~Hz},-\mathrm{CH}=\mathrm{N}), 8.17(\mathrm{dd}, 2 \mathrm{H}, J=$ 1.80, 3.6 Hz, Ar-H), 7.75 (d, 1H, $J=8.40 \mathrm{~Hz}, \mathrm{Ar}-\mathrm{H}), 7.63-$ 7.59 (m, 3H, Ar-H), 7.56 (t, 1H, J= $7.80 \mathrm{~Hz}$, Ar-H), 7.44 (t, $1 \mathrm{H}, J=7.80 \mathrm{~Hz}, \mathrm{Ar}-\mathrm{H}) ;{ }^{13} \mathrm{C}$ NMR $\left(75 \mathrm{MHz}, \mathrm{DMSO}-d_{6}\right): \delta$ $171.25,168.37,141.34,132.01,130.19,129.63,127.72$, 127.57, 126.55, 123.73, 121.73, 120.89, 111.73; ESI-MS m/z $263(\mathrm{M}+\mathrm{H})^{+}$.

3-(3-Methylphenyl)-5-(3-indazolyl)-1,2,4-oxadiazole (3c): Compound $\mathbf{3 c}$ was prepared from compound $\mathbf{3}$ and amidoxime 2c following general procedure-IV method-A $(80 \%$ yield). Cream color solid; m.p.: $215-218{ }^{\circ} \mathrm{C}$; IR (KBr, $\mathrm{v}_{\max }$, $\left.\mathrm{cm}^{-1}\right): 3205(\mathrm{NH}), 2943,1601(\mathrm{C}=\mathrm{N}), 1336,1040,751 ;{ }^{1} \mathrm{H}$ NMR (300 MHz, DMSO- $\left.d_{6}\right): \delta 14.25$ (s, $\left.1 \mathrm{H},-\mathrm{NH}\right), 8.33$ (d, $1 \mathrm{H}, J=8.10 \mathrm{~Hz},-\mathrm{CH}=\mathrm{N}), 7.98(\mathrm{t}, 2 \mathrm{H}, J=4.6 \mathrm{~Hz}, \mathrm{Ar}-\mathrm{H}), 7.75$ (d, $1 \mathrm{H}, J=8.4 \mathrm{~Hz}, \mathrm{Ar}-\mathrm{H}), 7.57-7.40$ (m, 4H, Ar-H), 2.42 (s, $\left.3 \mathrm{H},-\mathrm{CH}_{3}\right) ;{ }^{13} \mathrm{C}$ NMR $\left(75 \mathrm{MHz}, \mathrm{DMSO}-d_{6}\right): \delta 171.14,168.41$, $141.31,138.99,132.60,129.46,127.93,127.69,126.47$, 124.71, 123.68, 121.71, 120.91, 111.68, 21.26; ESI-MS m/z $277(\mathrm{M}+\mathrm{H})^{+}$.

3-(3-Fluorophenyl)-5-(3-indazolyl)-1,2,4-oxadiazole (3d): Compound $\mathbf{3 d}$ was prepared from compound $\mathbf{3}$ and amidoxime 2d following general procedure-IV method-A (70 \% yield). Pale brown color solid; m.p.: $>200{ }^{\circ} \mathrm{C}$; IR (KBr, $\mathrm{v}_{\max }$, $\left.\mathrm{cm}^{-1}\right): 3196(\mathrm{NH}), 1910,1603(\mathrm{C}=\mathrm{N}), 1462,1335,1044,865$, 772; ${ }^{1} \mathrm{H}$ NMR (300 MHz, DMSO- $\left.d_{6}\right): \delta 14.27$ (s, $\left.1 \mathrm{H},-\mathrm{NH}\right)$, $8.34(\mathrm{~d}, 1 \mathrm{H}, J=8.10 \mathrm{~Hz},-\mathrm{CH}=\mathrm{N}), 8.01(\mathrm{~d}, 1 \mathrm{H}, J=7.80 \mathrm{~Hz}$, Ar-H), 7.94-7.89 (m, 1H, Ar-H), 7.75 (d, 1H, J=8.40 Hz, Ar$\mathrm{H})$, 7.71-7.63 (m, 1H, Ar-H), 7.57-7.40 (m, 3H, Ar-H); ${ }^{13} \mathrm{C}$ NMR (75 MHz, DMSO- $\left.d_{6}\right)$ : $\delta 171.44,167.51,167.48,164.31$, $161.07,141.31,131.98,131.87,130.04,128.75,128.63$, $127.72,123.75,121.73,120.90,119.07,118.79,114.39$, 114.07, 111.69; ESI-MS m/z, $281(\mathrm{M}+\mathrm{H})^{+}$.

3-Methyl-5-(4-indazolyl)-1,2,4-oxadiazole (4a): Compound $\mathbf{4 a}$ was prepared from compound $\mathbf{4}$ and amidoxime $\mathbf{2 a}$ following general procedure-IV method-A (75\% yield). Cream colour solid; m.p.: $195-197{ }^{\circ} \mathrm{C}$; IR $\left(\mathrm{KBr}, \mathrm{v}_{\max }, \mathrm{cm}^{-1}\right): 3141$ $(\mathrm{NH}), 1596(\mathrm{C}=\mathrm{N}), 1572,1341,1198,743 ;{ }^{1} \mathrm{H}$ NMR (300 MHz, DMSO- $\left.d_{6}\right): \delta 13.60(\mathrm{~s}, 1 \mathrm{H},-\mathrm{NH}), 8.50(\mathrm{~s}, 1 \mathrm{H},-\mathrm{CH}=\mathrm{N})$, 7.95 (dd, 1H, $J=7.95,0.6 \mathrm{~Hz}$, Ar-H), 7.89 (d, 1H, $J=8.4 \mathrm{~Hz}$, Ar-H), 7.57 (dd, 1H, J = 7.2, 8.1 Hz, Ar-H), 2.46 (s, 3H, $\left.-\mathrm{CH}_{3}\right) ;{ }^{13} \mathrm{C}$ NMR $\left(75 \mathrm{MHz}, \mathrm{DMSO}-d_{6}\right): \delta 174.59,168.01$, 140.78, 133.42, 126.34, 122.30, 119.74, 115.89, 115.50, 11.69; HRMS: $m / z(\mathrm{M}+\mathrm{H})$ calcd. for $\mathrm{C}_{10} \mathrm{H}_{8} \mathrm{~N}_{4} \mathrm{O}$ : 201.0776; Found $(\mathrm{M}+\mathrm{H})^{+}:$201.0774; ESI-MS $m / z, 201(\mathrm{M}+\mathrm{H})^{+}$.

3-Phenyl-5-(4-indazolyl)-1,2,4-oxadiazole (4b): Compound $\mathbf{4 b}$ was prepared from compound $\mathbf{4}$ and amidoxime $\mathbf{2 b}$ following general procedure-IV method-A (85\% yield). Light brown colour solid; m.p.: 192-194 ${ }^{\circ} \mathrm{C}$; IR $\left(\mathrm{KBr}, \mathrm{v}_{\max }, \mathrm{cm}^{-1}\right)$ : $3170(\mathrm{NH}), 1622(\mathrm{C}=\mathrm{N}), 1568,1361,1193,740 ;{ }^{1} \mathrm{H}$ NMR $\left(300 \mathrm{MHz}, \mathrm{CDCl}_{3}\right): \delta 10.56$ (bs, $\left.1 \mathrm{H},-\mathrm{NH}\right), 8.89$ (s, 1H, $-\mathrm{CH}=\mathrm{N}), 8.24(\mathrm{dd}, 2 \mathrm{H}, J=7.2,3.6 \mathrm{~Hz}, \mathrm{Ar}-\mathrm{H}), 8.15(\mathrm{~d}, 1 \mathrm{H}, J=$ 7.2, Ar-H), 7.78 (d, 1H, J=8.4 Hz, Ar-H), 7.60-7.54 (m, 4H, $\mathrm{Ar}-\mathrm{H}) ;{ }^{13} \mathrm{C}$ NMR $\left(75 \mathrm{MHz}, \mathrm{DMSO}-d_{6}\right): \delta 174.80,168.96$, $140.48,135.46,131.18,128.80,127.50,126.83,126.53$, 122.71, 120.44, 116.68, 114.37; HRMS: $m / z(\mathrm{M}+\mathrm{Na})$ calcd. for $\mathrm{C}_{15} \mathrm{H}_{10} \mathrm{~N}_{4} \mathrm{ONa}$ : 285.0752; Found $(\mathrm{M}+\mathrm{Na})^{+}: 285.0796$; ESI$\mathrm{MS} m / z, 263(\mathrm{M}+\mathrm{H})^{+}$.

3-(3-Methylphenyl)-5-(4-indazolyl)-1,2,4-oxadiazole (4c): Compound $4 c$ was prepared from compound 4 and 
amidoxime $2 \mathbf{c}$ following general procedure-IV method-A (80\% yield). Cream colour solid; IR $\left(\mathrm{KBr}, \mathrm{v}_{\max }, \mathrm{cm}^{-1}\right): 3118$ $(\mathrm{NH}), 1621(\mathrm{C}=\mathrm{N}), 1569,1361,961,750 ;{ }^{1} \mathrm{H}$ NMR $(300 \mathrm{MHz}$, DMSO- $\left.d_{6}\right): \delta 10.35$ (s, $\left.1 \mathrm{H},-\mathrm{NH}\right), 8.89(\mathrm{~s}, 1 \mathrm{H},-\mathrm{CH}=\mathrm{N}), 8.15$ (d, $1 \mathrm{H}, J=7.5 \mathrm{~Hz}, \mathrm{Ar}-\mathrm{H}), 8.05$ (d, $2 \mathrm{H}, J=9.0 \mathrm{~Hz}, \mathrm{Ar}-\mathrm{H}), 7.77$ $(\mathrm{d}, 1 \mathrm{H}, J=8.4 \mathrm{~Hz}, \mathrm{Ar}-\mathrm{H}), 7.57$ (dd, $1 \mathrm{H}, J=8.1,7.5 \mathrm{~Hz}, \mathrm{Ar}-\mathrm{H})$, 7.46-7.35 (m, 2H, Ar-H), $2.48\left(\mathrm{~s}, 3 \mathrm{H},-\mathrm{CH}_{3}\right) ;{ }^{13} \mathrm{C}$ NMR $(75$ MHz, DMSO- $\left.d_{6}\right): \delta 175.10,168.68,140.77,139.00,133.73$, $132.60,129.46,127.93,126.48,126.33,124.70,122.47$, 119.86, 116.09, 115.44, 21.25; HRMS: $m / z(\mathrm{M}+\mathrm{Na})$ calcd. for $\mathrm{C}_{16} \mathrm{H}_{12} \mathrm{~N}_{4} \mathrm{ONa}$ : 299.0909; Found $(\mathrm{M}+\mathrm{Na})^{+}: 299.0919$; ESIMS $m / z 277(\mathrm{M}+\mathrm{H})^{+}$.

3-(3-Fluorophenyl)-5-(4-indazolyl)-1,2,4-oxadiazole (4d): Compound $\mathbf{4 d}$ was prepared from compound $\mathbf{4}$ and amidoxime 2d following general procedure-IV method-A (70 \% yield). Half-white colour solid; IR (KBr, $\left.v_{\max }, \mathrm{cm}^{-1}\right): 3161(\mathrm{NH}), 1604$ $(\mathrm{C}=\mathrm{N}), 1566,1362,961,754 ;{ }^{1} \mathrm{H}$ NMR $(300 \mathrm{MHz}$, DMSO$\left.d_{6}\right): \delta 13.63(\mathrm{~s}, 1 \mathrm{H},-\mathrm{NH}), 8.70(\mathrm{~s}, 1 \mathrm{H},-\mathrm{CH}=\mathrm{N}), 8.04(\mathrm{t}, 2 \mathrm{H}$, $J=4.5 \mathrm{~Hz}, \mathrm{Ar}-\mathrm{H}), 7.95$ (d, 2H, $J=8.5 \mathrm{~Hz}, \mathrm{Ar}-\mathrm{H}), 7.70-7.57$ (m, 2H, Ar-H), 7.52-7.45 (m, 1H, Ar-H); ${ }^{13} \mathrm{C}$ NMR $(75 \mathrm{MHz}$, DMSO- $\left.d_{6}\right): \delta 175.34,167.69,167.66,164.25,161.01,140.81$, $133.58,131.83,131.72,129.71,128.60,126.19,123.65$, $123.61,122.48,119.78,118.96,118.68,116.25,115.18$, 114.31, 114.00; HRMS: $m / z$ (M) calcd. for $\mathrm{C}_{15} \mathrm{H}_{9} \mathrm{~N}_{4} \mathrm{OF}$ : 280.2636; Found $(\mathrm{M})^{+}$: Not ionized; ESI-MS $\mathrm{m} / \mathrm{z}, 281$ $(\mathrm{M}+\mathrm{H})^{+}$.

3-Methyl-5-(5-indazolyl)-1,2,4-oxadiazole (5a): Compound $\mathbf{5 a}$ was prepared from compound $\mathbf{5}$ and amidoxime $\mathbf{2 a}$ following general procedure-IV method-A (65\% yield). Light yellow colour solid; m.p.: 204-205 ${ }^{\circ} \mathrm{C}$; IR $\left(\mathrm{KBr}, \mathrm{v}_{\max }, \mathrm{cm}^{-1}\right)$ : $3143(\mathrm{NH}), 2924,1626(\mathrm{C}=\mathrm{N}), 1392,952,755 ;{ }^{1} \mathrm{H}$ NMR $(300$ MHz, DMSO- $\left.d_{6}\right): \delta 13.49(\mathrm{~s}, 1 \mathrm{H},-\mathrm{NH}), 8.58(\mathrm{~s}, 1 \mathrm{H},-\mathrm{CH}=\mathrm{N})$, 8.27 (s, 1H, Ar-H), 8.00 (dd, 1H, $J=8.7,1.5 \mathrm{~Hz}$, Ar-H), 7.71 $(\mathrm{d}, 1 \mathrm{H}, J=8.7 \mathrm{~Hz}, \mathrm{Ar}-\mathrm{H}), 2.39$ (s, 3H, $\left.-\mathrm{CH}_{3}\right) ;{ }^{13} \mathrm{C}$ NMR $(75$ MHz, DMSO- $\left.d_{6}\right): \delta 175.71,167.85,141.67,135.70,125.23$, 123.25, 122.48, 116.19, 111.71, 11.63; ESI-MS m/z $201(\mathrm{M}+\mathrm{H})^{+}$.

3-Phenyl-5-(5-indazolyl)-1,2,4-oxadiazole (5b): Compound $\mathbf{5 b}$ was prepared from compound $\mathbf{5}$ and amidoxime $\mathbf{2 b}$ following general procedure-IV method-A (75\% yield). Cream colour solid; m.p.: 204-205 ${ }^{\circ}$ C; IR $\left(\mathrm{KBr}, \mathrm{v}_{\max }, \mathrm{cm}^{-1}\right): 3144$ (NH), 2928, $1568(\mathrm{C}=\mathrm{N}), 1369,954,752 ;{ }^{1} \mathrm{H}$ NMR $(300 \mathrm{MHz}$, DMSO- $\left.d_{6}\right): \delta 13.53(\mathrm{~s}, 1 \mathrm{H},-\mathrm{NH}), 8.70(\mathrm{~s}, 1 \mathrm{H},-\mathrm{CH}=\mathrm{N}), 8.32$ (s, 1H, Ar-H), 8.13-8.08 (m, 3H, Ar-H), 7.77 (d, 1H, $J=8.8$ $\mathrm{Hz}, \mathrm{Ar}-\mathrm{H}), 7.61-7.57$ (m, 3H, Ar-H); ${ }^{13} \mathrm{C}$ NMR (75 MHz, DMSO- $\left.d_{6}\right): \delta 176.46,168.53,141.78,135.83,131.92,129.60$, $127.45,126.72,125.40,123.31,122.86,116.05,111.79$; ESIMS $m / z 263(\mathrm{M}+\mathrm{H})^{+}$.

3-(3-Methylphenyl)-5-(5-indazolyl)-1,2,4-oxadiazole (5c): Compound $\mathbf{5 c}$ was prepared from compound $\mathbf{5}$ and amidoxime 2c following general procedure-IV method-A (70\% yield). Off-white colour solid; IR ( $\left.\mathrm{KBr}, \mathrm{v}_{\max }, \mathrm{cm}^{-1}\right): 3146$ (NH), 2926, 1627, $1603(\mathrm{C}=\mathrm{N}), 1370,955,756 ;{ }^{1} \mathrm{H}$ NMR $(300$ MHz, DMSO- $\left.d_{6}\right): \delta 13.53(\mathrm{~s}, 1 \mathrm{H},-\mathrm{NH}), 8.70(\mathrm{~s}, 1 \mathrm{H},-\mathrm{CH}=\mathrm{N})$, 8.32 (s, 1H, Ar-H), 8.11(d, 1H, J = 8.8 Hz, Ar-H), 7.89 (d, 2H, $J=12.1 \mathrm{~Hz}, \mathrm{Ar}-\mathrm{H}), 7.76(\mathrm{~d}, 1 \mathrm{H}, J=8.7 \mathrm{~Hz}, \mathrm{Ar}-\mathrm{H}), 7.49-7.40$ (m, 2H, Ar-H), 2.46 (s, 3H, - $\left.\mathrm{CH}_{3}\right) ;{ }^{13} \mathrm{C}$ NMR (75 MHz, DMSO$\left.d_{6}\right): \delta 176.37,168.57,141.79,138.97,135.83,132.52,129.47$, $127.85,126.66,125.39,124.59,123.32,122.83,116.07$, 111.76, 21.25; ESI-MS $m / z, 277(\mathrm{M}+\mathrm{H})^{+}$.
3-(3-Fluorophenyl)-5-(5-indazolyl)-1,2,4-oxadiazole (5d): Compound $\mathbf{5 d}$ was prepared from compound $\mathbf{5}$ and amidoxime 2d following general procedure-IV method-A (60 $\%$ yield). Light yellow colour solid; IR $\left(\mathrm{KBr}, v_{\max }, \mathrm{cm}^{-1}\right): 3143$ (NH), 2928, $1626(\mathrm{C}=\mathrm{N}), 1457,956,758 ;{ }^{1} \mathrm{H} \mathrm{NMR}(300 \mathrm{MHz}$, DMSO- $\left.d_{6}\right): \delta 13.54(\mathrm{~s}, 1 \mathrm{H},-\mathrm{NH}), 8.71(\mathrm{~s}, 1 \mathrm{H},-\mathrm{CH}=\mathrm{N}), 8.32$ (s, 1H, Ar-H), 8.09 (t, 1H, J=8.76, Ar-H), 7.94 (d, 1H, $J=7.8$ $\mathrm{Hz}, \mathrm{Ar}-\mathrm{H})$, 7.85-7.76 (m, 2H, Ar-H), 7.69-7.62 (m, 1H, Ar$\mathrm{H}), 7.50-7.44$ (m, 1H, Ar-H); ${ }^{13} \mathrm{C}$ NMR (75 MHz, DMSO- $\left.d_{6}\right)$ : $\delta$ 176.75, 167.67, 164.30, 161.06, 141.84, 135.88, 132.04, $131.93,128.96,128.84,125.42,123.69,123.66,123.32$, $122.99,119.03,118.75,115.87,114.27,113.96,111.83$; ESI$\mathrm{MS} m / z, 281(\mathrm{M}+\mathrm{H})^{+}$.

3-Methyl-5-(6-indazolyl)-1,2,4-oxadiazole (6a): Compound $\mathbf{6 a}$ was prepared from compound $\mathbf{6}$ and amidoxime $\mathbf{2 a}$ following general procedure-IV method-A (85\% yield). Light yellow colour solid; IR (KBr, $\left.v_{\max }, \mathrm{cm}^{-1}\right): 3211(\mathrm{NH}), 1591$ $(\mathrm{C}=\mathrm{N}), 1571,1333,940,774 ;{ }^{1} \mathrm{H}$ NMR (300 MHz, DMSO$\left.d_{6}\right): \delta 13.51(\mathrm{~s}, 1 \mathrm{H},-\mathrm{NH}), 8.22(\mathrm{~d}, 2 \mathrm{H}, J=7.4 \mathrm{~Hz},-\mathrm{CH}=\mathrm{N})$, 7.98 (d, 1H, $J=8.4 \mathrm{~Hz}, \mathrm{Ar}-\mathrm{H}), 7.77$ (dd, $1 \mathrm{H}, J=8.4,1.2 \mathrm{~Hz}$, Ar-H), $2.41\left(\mathrm{~s}, 3 \mathrm{H},-\mathrm{CH}_{3}\right) ;{ }^{13} \mathrm{C}$ NMR (75 MHz, DMSO- $\left.d_{6}\right): \delta$ 175.58, 168.00, 139.64, 134.35, 125.47, 122.28, 121.08, 119.27, 110.73, 11.60; HRMS: $m / z(\mathrm{M}+\mathrm{H})$ calcd. for $\mathrm{C}_{10} \mathrm{H}_{8} \mathrm{~N}_{4} \mathrm{O}$ : 201.0776; Found $(\mathrm{M}+\mathrm{H})^{+}$: 201.0766; ESI-MS $\mathrm{m} / \mathrm{z} 201(\mathrm{M}+\mathrm{H})^{+}$.

3-Phenyl-5-(6-indazolyl)-1,2,4-oxadiazole (6b): Compound $\mathbf{6 b}$ was prepared from compound $\mathbf{6}$ and amidoxime $\mathbf{2 b}$ following general procedure-IV method-A (90\% yield). Brown colour solid; IR $\left(\mathrm{KBr}, v_{\max }, \mathrm{cm}^{-1}\right)$ : $3294(\mathrm{NH}), 1597$ $(\mathrm{C}=\mathrm{N}), 1445,1361,742,684 ;{ }^{1} \mathrm{H}$ NMR $(300 \mathrm{MHz}$, DMSO$\left.d_{6}\right): \delta 13.56(\mathrm{~s}, 1 \mathrm{H},-\mathrm{NH}), 8.37(\mathrm{~s}, 1 \mathrm{H},-\mathrm{CH}=\mathrm{N}), 8.26(\mathrm{~s}, 1 \mathrm{H}$, Ar-H), $8.11(\mathrm{t}, 1 \mathrm{H}, J=4.8 \mathrm{~Hz}, \mathrm{Ar}-\mathrm{H}), 8.03(\mathrm{~d}, 1 \mathrm{H}, J=8.4 \mathrm{~Hz}$, Ar-H), 7.88 (d, 1H, $J=8.1 \mathrm{~Hz}, \mathrm{Ar}-\mathrm{H}), 7.61$ (t, 4H, $J=3.9$, Ar$\mathrm{H}) ;{ }^{13} \mathrm{C}$ NMR (75 MHz, DMSO- $\left.d_{6}\right): \delta 176.35,168.66,139.63$, $134.41,132.01,129.62,127.48,126.57,125.62,122.42$, 120.98, 119.45, 111.11; ESI-MS m/z $263(\mathrm{M}+\mathrm{H})^{+}$.

3-(3-Methylphenyl)-5-(6-indazolyl)-1,2,4-oxadiazole (6c): Compound $\mathbf{6 c}$ was prepared from compound $\mathbf{6}$ and amidoxime 2c following general procedure-IV method-A (75\% yield). Off-white colour solid; m.p.: $>220{ }^{\circ} \mathrm{C}$; IR (KBr, $\mathrm{v}_{\max }$, $\left.\mathrm{cm}^{-1}\right): 3434(\mathrm{NH}), 1597(\mathrm{C}=\mathrm{N}), 1572,1354,754 ;{ }^{1} \mathrm{H}$ NMR (300 MHz, DMSO-d $\left.d_{6}\right): \delta 13.5(\mathrm{~s}, 1 \mathrm{H},-\mathrm{NH}), 8.36(\mathrm{~s}, 1 \mathrm{H}$, $-\mathrm{CH}=\mathrm{N}), 8.25(\mathrm{~s}, 1 \mathrm{H}, \mathrm{Ar}-\mathrm{H}), 8.02(\mathrm{~d}, 1 \mathrm{H}, J=8.4 \mathrm{~Hz}, \mathrm{Ar}-\mathrm{H})$, 7.93-7.86 (m, 3H, Ar-H), 7.51-7.41 (m, 2H, Ar-H), 2.41 (s, $\left.3 \mathrm{H},-\mathrm{CH}_{3}\right) ;{ }^{13} \mathrm{C}$ NMR $\left(75 \mathrm{MHz}, \mathrm{DMSO}-d_{6}\right): \delta 176.25,168.70$, $139.67,139.01,134.41,132.62,129.50,127.86,126.48$, 125.61, 124.63, 122.40, 121.00, 119.45, 111.05, 21.25; HRMS: $m / z(\mathrm{M}+\mathrm{H})$ calcd. for $\mathrm{C}_{16} \mathrm{H}_{12} \mathrm{~N}_{4} \mathrm{O}$ : 277.1089; Found $(\mathrm{M}+\mathrm{H})^{+}:$277.1265; ESI-MS m/z, $277(\mathrm{M}+\mathrm{H})^{+}$.

3-(3-Fluorophenyl)-5-(6-indazolyl)-1,2,4-oxadiazole (6d): Compound $\mathbf{6} \mathbf{d}$ was prepared from compound $\mathbf{6}$ and amidoxime 2d following general procedure-IV method-A (72\% yield). Cream colour solid; m.p.: $>220^{\circ} \mathrm{C}$; IR $\left(\mathrm{KBr}, \nu_{\max }, \mathrm{cm}^{-1}\right)$ : $3444(\mathrm{NH}), 2868,1601(\mathrm{C}=\mathrm{N}), 1570,1365,956,755 ;{ }^{1} \mathrm{H} \mathrm{NMR}$ (300 MHz, DMSO- $\left.d_{6}\right): \delta 13.58(\mathrm{~s}, 1 \mathrm{H},-\mathrm{NH}), 8.37$ (s, 1H, $-\mathrm{CH}=\mathrm{N}), 8.26$ (s, 1H, Ar-H), 8.02 (t, $1 \mathrm{H}, J=8.1 \mathrm{~Hz}, \mathrm{Ar}-\mathrm{H})$, 7.97-7.94 (m, 2H, Ar-H), 7.89-7.82 (m, 2H, Ar-H), 7.69-7.62 (m, 1H, Ar-H), 7.51-7.45 (m, 1H, Ar-H); ${ }^{13} \mathrm{C} \mathrm{NMR}(75 \mathrm{MHz}$, DMSO- $\left.d_{6}\right)$ : $\delta 176.57,167.78,167.74,164.27,161.02,139.70$, 
$134.32,131.98,131.87,128.74,128.63,125.66,123.67$, $123.63,122.40,120.76,119.41,119.06,118.78,114.26$, 113.95, 111.23; ESI-MS m/z $281(\mathrm{M}+\mathrm{H})^{+}$

3-Methyl-5-(7-indazolyl)-1,2,4-oxadiazole (7a): Compound 7a was prepared from compound 28a following general procedure-III (78 \% yield). Cream colour solid; m.p.: $>220$ ${ }^{\circ} \mathrm{C}$; IR $\left(\mathrm{KBr}, v_{\max }, \mathrm{cm}^{-1}\right): 3266(\mathrm{NH}), 1622(\mathrm{C}=\mathrm{N}), 1325,949$, 747; ${ }^{1} \mathrm{H}$ NMR $\left(300 \mathrm{MHz}, \mathrm{CDCl}_{3}\right): \delta 13.38$ (s, 1H, -NH), 8.29 $(\mathrm{d}, 1 \mathrm{H}, J=1.38,-\mathrm{CH}=\mathrm{N}), 8.14(\mathrm{~d}, 2 \mathrm{H}, J=7.32$, Ar-H), 7.35 $(\mathrm{q}, 1 \mathrm{H}, J=7.47, \mathrm{Ar}-\mathrm{H}), 2.46\left(\mathrm{~s}, 3 \mathrm{H},-\mathrm{CH}_{3}\right) ;{ }^{13} \mathrm{C}$ NMR $(75$ MHz, DMSO- $\left.d_{6}\right): \delta 173.47,167.87,136.31,135.21,127.20$, 126.87, 125.07, 120.90, 106.60, 11.67; ESI-MS m/z 201 $(\mathrm{M}+\mathrm{H})^{+}$.

3-Phenyl-5-(7-indazolyl)-1,2,4-oxadiazole (7b): Compound $\mathbf{7 b}$ was prepared from compound $\mathbf{2 8 b}$ following to general procedure-III (81 \% yield). Cream coloured solid; m.p.: $>220{ }^{\circ} \mathrm{C}$; IR $\left(\mathrm{KBr}, v_{\max }, \mathrm{cm}^{-1}\right): 3314(\mathrm{NH}), 1621(\mathrm{C}=\mathrm{N}), 1338$, 946, 741; ${ }^{1} \mathrm{H}$ NMR $\left(300 \mathrm{MHz}, \mathrm{CDCl}_{3}\right): \delta 13.55$ (s, $\left.1 \mathrm{H},-\mathrm{NH}\right)$, $8.39(\mathrm{t}, 2 \mathrm{H}, J=4.50 \mathrm{~Hz}, \mathrm{Ar}-\mathrm{H}), 8.35(\mathrm{~s}, 1 \mathrm{H},-\mathrm{CH}=\mathrm{N}), 8.22$ $(\mathrm{dd}, 2 \mathrm{H}, J=7.5,3.9 \mathrm{~Hz}, \mathrm{Ar}-\mathrm{H}), 7.61(\mathrm{t}, 3 \mathrm{H}, J=3.60 \mathrm{~Hz}, \mathrm{Ar}-$ $\mathrm{H}), 7.39$ (t, $1 \mathrm{H}, J=7.50 \mathrm{~Hz}, \mathrm{Ar}-\mathrm{H}) ;{ }^{13} \mathrm{C} \mathrm{NMR}(75 \mathrm{MHz}, \mathrm{DMSO}-$ $\left.d_{6}\right): \delta 173.75,168.68,136.32,135.32,132.04,129.42,128.07$, 127.21, 126.50, 125.16, 120.97, 106.64; ESI-MS m/z 263 $(\mathrm{M}+\mathrm{H})^{+}$.

3-(3-Methylphenyl)-5-(7-indazolyl)-1,2,4-oxadiazole (7c): Compound 7c was prepared from compound 28c following general procedure-III (76 \% yield). Light brown color solid; m.p.: $>220{ }^{\circ} \mathrm{C}$; IR (KBr, $\left.v_{\max }, \mathrm{cm}^{-1}\right)$ : $3291(\mathrm{NH}), 1622$ $(\mathrm{C}=\mathrm{N}), 1463,1332,753 ;{ }^{1} \mathrm{H}$ NMR $\left(300 \mathrm{MHz}, \mathrm{DMSO}-d_{6}\right): \delta$ 13.52 (s, 1H, -NH), 8.36 (s, 1H, - $\mathrm{CH}=\mathrm{N}), 8.24-8.17$ (m, 4H, Ar-H), 7.50-7.35 (m, 3H, Ar-H), 2.48 (s, 3H, $\left.-\mathrm{CH}_{3}\right) ;{ }^{13} \mathrm{C} \mathrm{NMR}$ $\left(75 \mathrm{MHz}, \mathrm{DMSO}-d_{6}\right): \delta 173.61,168.71,138.84,136.30$, $135.32,132.60,129.26,128.52,127.16,125.11,120.93$, 106.54, 21.26; ESI-MS m/z $277(\mathrm{M}+\mathrm{H})^{+}$.

3-(3-Fluorophenyl)-5-(7-indazolyl)-1,2,4-oxadiazole (7d): Compound $\mathbf{7 d}$ was prepared from compound $\mathbf{2 8 d}$ following general procedure-III (72\% yield). Brown color solid; m.p.: $>220^{\circ} \mathrm{C}$; IR $\left(\mathrm{KBr}, v_{\max }, \mathrm{cm}^{-1}\right): 3273(\mathrm{NH}), 1623(\mathrm{C}=\mathrm{N})$, 1340, 952, 755; ${ }^{1} \mathrm{H}$ NMR (300 MHz, DMSO- $\left.d_{6}\right): \delta 13.59$ (s, $1 \mathrm{H},-\mathrm{NH}), 8.43-8.38(\mathrm{~m}, 1 \mathrm{H},-\mathrm{CH}=\mathrm{N}), 8.36(\mathrm{~d}, 1 \mathrm{H}, J=1.20$ $\mathrm{Hz}, \mathrm{Ar}-\mathrm{H}), 8.24-8.15$ (m, 3H, Ar-H), 7.68-7.61 (m, 1H, ArH), 7.51-7.44 (m, 1H, Ar-H), 7.40 (t, 1H, $J=8.10 \mathrm{~Hz}, \mathrm{Ar}-\mathrm{H})$; ${ }^{13} \mathrm{C}$ NMR $\left(75 \mathrm{MHz}, \mathrm{DMSO}-d_{6}\right): \delta 173.82,167.83,164.51$, $161.28,136.25,135.33,131.70,131.59,128.74,128.62$, $127.37,127.15,125.15,123.82,123.78,120.95,119.03$, 118.75, 115.54, 115.23, 106.34; ESI-MS m/z $281(\mathrm{M}+\mathrm{H})^{+}$.

3-Methyl-5-(2-nitro-3-methylphenyl)-1,2,4-oxadiazole (27a): Compound 27a was prepared from compound 24 and amidoxime 2a following general procedure-IV method-A (72\% yield): ${ }^{1} \mathrm{H}$ NMR (300 MHz, DMSO- $\left.d_{6}\right) \delta 8.04(\mathrm{dd}, 1 \mathrm{H}$, $J=0.96,6.63 \mathrm{~Hz}, \mathrm{Ar}-\mathrm{H}), 7.83-7.71$ (m, 2H, Ar-H), 2.39 (s, $\left.3 \mathrm{H},-\mathrm{CH}_{3}\right), 2.33\left(\mathrm{~s}, 3 \mathrm{H},-\mathrm{CH}_{3}\right)$; ESI-MS $m / z 220(\mathrm{M}+\mathrm{H})^{+}$.

3-Phenyl-5-(2-nitro-3-methylphenyl)-1,2,4-oxadiazole (27b): Compound 27b was prepared from compound 24 and amidoxime $\mathbf{2 b}$ following general procedure-IV method-A (79 \% yield): ${ }^{1} \mathrm{H}$ NMR $\left(300 \mathrm{MHz}, \mathrm{DMSO}-d_{6}\right) \delta 8.15(\mathrm{dd}, 1 \mathrm{H}$, $J=0.96,6.54 \mathrm{~Hz}, \mathrm{Ar}-\mathrm{H}$ ), 8.01-.98 (m, 2H, Ar-H), 7.87-7.75 (m, 2H, Ar-H), 7.61-7.58 (m, 3H, Ar-H), 2.36 (s, 3H, $\left.-\mathrm{CH}_{3}\right)$; ESI-MS $m / z, 282(\mathrm{M}+\mathrm{H})^{+}$.
3-(3-Methyl-phenyl)-5-(2-nitro-3-methylphenyl)-1,2,4oxadiazole (27c): Compound $27 \mathrm{c}$ was prepared from compound $\mathbf{2 4}$ and amidoxime $\mathbf{2 c}$ according to general procedureIV method-A (80\% yield): ${ }^{1} \mathrm{H}$ NMR (300 MHz, DMSO- $\left.d_{6}\right) \delta$ 8.15 (dd, $1 \mathrm{H}, J=0.99,6.51 \mathrm{~Hz}, \mathrm{Ar}-\mathrm{H}), 7.87-7.75$ (m, 4H, Ar$\mathrm{H}), 7.46$ (dd, 2H, J = 7.02, $0.60 \mathrm{~Hz}, \mathrm{Ar}-\mathrm{H}), 2.39$ (s, 3H, $-\mathrm{CH}_{3}$ ), $2.37\left(\mathrm{~s}, 3 \mathrm{H},-\mathrm{CH}_{3}\right)$; ESI-MS $m / z 300(\mathrm{M}+\mathrm{H})^{+}$.

3-(3-Fluoro-phenyl)-5-(2-nitro-3-methylphenyl)-1,2,4oxadiazole (27d): Compound 27d was prepared from compound $\mathbf{2 4}$ and amidoxime $\mathbf{2 d}$ following general procedure-IV method-A (71\% yield): ${ }^{1} \mathrm{H}$ NMR $\left(300 \mathrm{MHz}\right.$, DMSO- $\left.d_{6}\right) \delta$ 8.16 (dd, $1 \mathrm{H}, J=0.93,6.66 \mathrm{~Hz}, \mathrm{Ar}-\mathrm{H}), 7.88-7.62$ (m, 5H, Ar$\mathrm{H})$, 7.53-7.49 (m, 1H, Ar-H), 2.37 (s, 3H, - $\mathrm{CH}_{3}$ ); ESI-MS m/z $300(\mathrm{M}+\mathrm{H})^{+}$.

3-Methyl-5-(2-amino-3-methylphenyl)-1,2,4-oxadiazole (28a): Compound 28a was prepared from compound 27a following general procedure-II (79 \% yield): ${ }^{1} \mathrm{H}$ NMR $\left(300 \mathrm{MHz}, \mathrm{DMSO}-d_{6}\right) \delta 8.02(\mathrm{dd}, 1 \mathrm{H}, J=6.96,0.63 \mathrm{~Hz}$, Ar-H), 7.81-7.69 (m, 2H, Ar-H), 2.38 (s, 3H,-CH $), 2.32$ (s, $\left.3 \mathrm{H},-\mathrm{CH}_{3}\right)$; ESI-MS m/z $190(\mathrm{M}+\mathrm{H})^{+}$

3-Phenyl-5-(2-amino-3-methylphenyl)-1,2,4-oxadiazole (28b): Compound 28b was prepared from compound 27b following general procedure-II (86 \% yield): ${ }^{1} \mathrm{H}$ NMR (300 $\left.\mathrm{MHz}, \mathrm{DMSO}-d_{6}\right) \delta$ 8.11-8.07 (m, 2H, Ar-H), $7.75(\mathrm{dd}, 1 \mathrm{H}$, $J=6.99,1.14 \mathrm{~Hz}$, Ar-H), 7.62-7.57 (m, 3H, Ar-H), $7.27(\mathrm{t}$, $1 \mathrm{H}, J=6.63 \mathrm{~Hz}, \mathrm{Ar}-\mathrm{H}), 6.67$ (t, 1H, $J=7.89 \mathrm{~Hz}, \mathrm{Ar}-\mathrm{H}), 2.19$ (s, 3H, $\left.-\mathrm{CH}_{3}\right)$; ESI-MS $m / z, 252(\mathrm{M}+\mathrm{H})^{+}$.

3-(3-Methyl-phenyl)-5-(2-amino-3-methylphenyl)1,2,4-oxadiazole (28c): Compound 28c was prepared from compound 27c following general procedure-II (89\% yield): ${ }^{1} \mathrm{H}$ NMR $\left(300 \mathrm{MHz}, \mathrm{DMSO}-d_{6}\right) \delta 7.88(\mathrm{~d}, 2 \mathrm{H}, J=8.94 \mathrm{~Hz}$, Ar-H), 7.76 (dd, 1H, $J=7.05,1.05 \mathrm{~Hz}, \mathrm{Ar}-\mathrm{H}), 7.50-7.40$ (m, $2 \mathrm{H}, \mathrm{Ar}-\mathrm{H}), 7.27$ (t, 1H, $J=5.73 \mathrm{~Hz}, \mathrm{Ar}-\mathrm{H}), 6.66$ (t, 1H, $J=$ $7.83 \mathrm{~Hz}, \mathrm{Ar}-\mathrm{H}), 6.12$ (s, 2H, -NH $\mathrm{NH}_{2}, 2.47$ (s, 3H, - $\left.\mathrm{CH}_{3}\right), 2.19$ $(\mathrm{s}, 3 \mathrm{H})$; ESI-MS $m / z, 266(\mathrm{M}+\mathrm{H})^{+}$.

3-(3-Fluoro-phenyl)-5-(2-amino-3-methylphenyl)1,2,4-oxadiazole (28d): Compound 28d was prepared from compound 27d following general procedure-IV method-A (80 \% yield): ${ }^{1} \mathrm{H}$ NMR (300 MHz, DMSO- $\left.d_{6}\right) \delta$ 7.95-7.85 (m, $2 \mathrm{H}, \mathrm{Ar}-\mathrm{H}), 7.73$ (t, 1H, $J=7.14,0.87 \mathrm{~Hz}, \mathrm{Ar}-\mathrm{H}), 7.68-7.60$ (m, 1H, Ar-H), 7.50-7.43 (m, 1H, Ar-H), 7.26 (d, 1H, J = 6.90 $\mathrm{Hz}, \mathrm{Ar}-\mathrm{H}), 6.73$ (s, 2H, $\left.-\mathrm{NH}_{2}\right), 6.65$ (t, 1H, $J=7.86,7.41 \mathrm{~Hz}$, Ar-H), 2.18 (s, 3H).; ESI-MS m/z $270(\mathrm{M}+\mathrm{H})^{+}$.

Synthesis of compound 10: To a solution of phenyl hydrazine $(\mathbf{8})(92.5 \mathrm{mmol})$ in water $(100.0 \mathrm{~mL})$ 4-bromobenzaldehyde (9) (92.5 mmol) was added slowly over a period of $1 \mathrm{~h}$ at room temperature. The solution was stirred for $2 \mathrm{~h}$ at same temperature and reaction mixture was cooled to $15^{\circ} \mathrm{C}$. The obtained solid was filtered and washed with isopropyl alcohol. The product was dryied at $50{ }^{\circ} \mathrm{C}$ under reduced pressure to get compound (10) with $95 \%$ yield. ${ }^{1} \mathrm{H}$ NMR (300 MHz, DMSO- $\left.d_{6}\right) \delta 10.42(\mathrm{~s}, 1 \mathrm{H}), 7.79(\mathrm{~s}, 1 \mathrm{H}), 7.59-7.52(\mathrm{~m}$, 4H), 7.22-7.7.17 (m, 2H), 7.06-7.03 (m, 2H), 6.75 (t, 1H, J = $7.26 \mathrm{~Hz})$; ESI-MS $m / z, 277(\mathrm{M}+2)^{+}$.

Synthesis of compound 12: To a solution of oxalyl chloride (76.0 mmol) in dichloromethane $(76.0 \mathrm{~mL})$ was added compound $10(69.0 \mathrm{mmol})$ in dichloromethane $(152.0 \mathrm{~mL})$ slowly over a period of $1 \mathrm{~h}$ at reflux temperature. This solution was stirred for $3 \mathrm{~h}$ at same temperature and reaction mixture was cooled 
to $25^{\circ} \mathrm{C}$. Reaction mixture was concentrated to dryness to get residue. The residue was dissolved in dichloromethane $(76 \mathrm{~mL})$ and aluminum chloride $(165.7 \mathrm{mmol})$ was added slowly to the reaction mixture maintaining the temperature below $25^{\circ} \mathrm{C}$. After complete addition the reaction mixture was refluxed for $3 \mathrm{~h}$ and reaction progress was monitored by TLC. After completion, reaction mixture was cooled to $0{ }^{\circ} \mathrm{C}$ and diluted with chilled water $(152 \mathrm{~mL})$. The reaction was further stirred for $1 \mathrm{~h}$ and organic layer was separated. Aqueous layer was extracted with dichloromethane and combined organic layers were washed with dil. $\mathrm{HCl}$ and brine. Organic layer was concentrated to dryness under reduced pressure to give compound 12 (91\% yield). Light brown colour solid; ${ }^{1} \mathrm{H}$ NMR (300 MHz, DMSO$\left.d_{6}\right) \delta 9.60(\mathrm{~s}, 1 \mathrm{H}), 7.87(\mathrm{~d}, 2 \mathrm{H}, J=8.49 \mathrm{~Hz}), 7.75-7.69(\mathrm{~m}$, $3 \mathrm{H}), 7.61(\mathrm{~d}, 1 \mathrm{H}, J=6.81 \mathrm{~Hz}), 7.45(\mathrm{~d}, 1 \mathrm{H}, J=7.89 \mathrm{~Hz}), 7.24-$ $7.20(\mathrm{~m}, 1 \mathrm{H})$.

Synthesis of compound 13: A solution of compound 12 $(13.7 \mathrm{mmol})$ in acetic acid $(45.0 \mathrm{~mL})$ and conc. hydrochloric acid $(6.6 \mathrm{~mL})$ was heated to 90 to $100{ }^{\circ} \mathrm{C}$ for $1 \mathrm{~h}$. Reaction mixture was concentrated under reduced pressure. Acetic acid (45 mL) was added to the residue and heated to $115^{\circ} \mathrm{C}$ for $1 \mathrm{~h}$ and cooled to room temperature and stirred for $1 \mathrm{~h}$. The solid obtained was filtered and washed with acetic acid followed by water. The product was dryied at $60{ }^{\circ} \mathrm{C}$ under reduced pressure to give compound $\mathbf{1 3}$ (85\% yield). Light yellow colour solid, m.p.: $>220{ }^{\circ} \mathrm{C} ;{ }^{1} \mathrm{H}$ NMR $\left(300 \mathrm{MHz}, \mathrm{DMSO}-d_{6}\right)$ $\delta 13.77$ (s, $1 \mathrm{H},-\mathrm{NH}), 12.0$ (s, 1H, $-\mathrm{COOH}), 8.05$ (d, $1 \mathrm{H}, J=$ $8.16 \mathrm{~Hz}, \mathrm{Ar}-\mathrm{H}), 7.62$ (d, $1 \mathrm{H}, J=8.43 \mathrm{~Hz}, \mathrm{Ar}-\mathrm{H}), 7.43-7.38$ (m, 1H, Ar-H), 7.28-7.23 (m, 1H, Ar-H).; ESI-MS m/z 177 $(\mathrm{M}+\mathrm{H})^{+}$.

Synthesis of compound 3: Compound 3 was prepared from 13 following general procedure-III (95\% yield, light yellow colour solid, m.p.: 160-165 $\left.{ }^{\circ} \mathrm{C}\right):{ }^{1} \mathrm{H}$ NMR $(300 \mathrm{MHz}$, DMSO- $\left.d_{6}\right) \delta 13.89$ (s, $\left.1 \mathrm{H},-\mathrm{NH}\right), 8.06(\mathrm{~d}, 1 \mathrm{H}, J=8.13 \mathrm{~Hz}, \mathrm{Ar}-$ H), 7.64 (d, 1H, J = 8.40 Hz, Ar-H), 7.46-7.40 (m, 1H, Ar-H), 7.32-7.26 (m, 1H, Ar-H), 3.90 (s, 3H, -CH $)$; ESI-MS m/z 177 $(\mathrm{M}+\mathrm{H})^{+}$.

\section{RESULTS AND DISCUSSION}

1,2,4-Oxadiazoles are most commonly synthesized from amidoximes and carboxylic acid derivatives in two steps. In the first step amidoximes were coupled with the activated acids to get O-acylamidoximes. In the second step intramolecular dehydrative cyclization results in 1,2,4-oxadiazoles ${ }^{9-11,24,27-29}$. These approaches generally require longer reaction times. $\mathrm{O}$-acylamidoximes were also prepared from amidoximes and were coupled with carboxylic acids in the presence of coupling reagents such as EDC, DCC or DIC. The cyclization reaction of $\mathrm{O}$-acylamidoximes is promoted either by heat or by bases such as $\mathrm{NaH}, \mathrm{NaOEt}$ or pyridine ${ }^{26}$. A recent report has been described by the use of tetrabutylammonium fluoride (TBAF) to promote the cyclization of $\mathrm{O}$-acylamidoximes in good yields ${ }^{27}$. Amidoximes were prepared form corresponding nitriles by treatment with hydroxylamine in methanol at room temperature. In an attempt to improve on these procedures, microwave-assisted 1,2,4-oxadiazole synthesis has been reported ${ }^{30}$ recently with moderate to good yields. Due to importance of 1,2,4-oxadiazoles and indazoles in the pharmaceutical area prompted us to synthesize 3-aryl/alkyl-5-indazolyl-1,2,4oxadiazoles (Scheme-I, Table-1) from amidoximes.

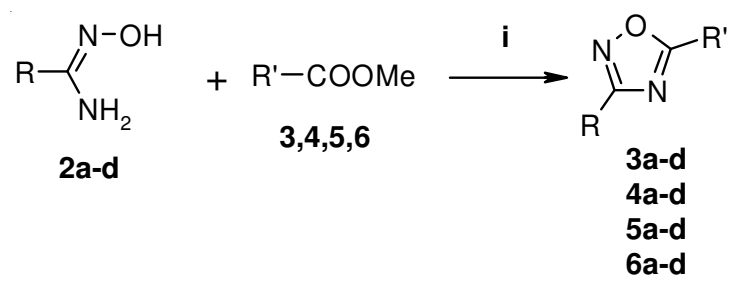

Scheme-I: General synthesis of 3,5-disubstituted oxadiazole; Conditions: (i) $\mathrm{K}_{2} \mathrm{CO}_{3}$, toluene, $110{ }^{\circ} \mathrm{C}$. ( $\mathrm{R}=\mathrm{Me}(2 \mathrm{a})$; $\mathrm{Ph}(2 \mathrm{~b}) ; 3-\mathrm{Me}-\mathrm{Ph}(2 \mathrm{c})$; 3 F-Ph (2d); $\mathrm{R}^{1}=3$-ind (3), 4-ind (4), 5-ind (5), 6-ind (6))

The precursor amidoximes $\mathbf{2 a - d}$ were synthesized from corresponding nitriles with hydroxylamine hydrochloride in the presence of base such as potassium carbonate in methanol by stirring at room temperature for $6 \mathrm{~h}$ to yield amidoximes in the range of $80-90 \%$ (Scheme-II).

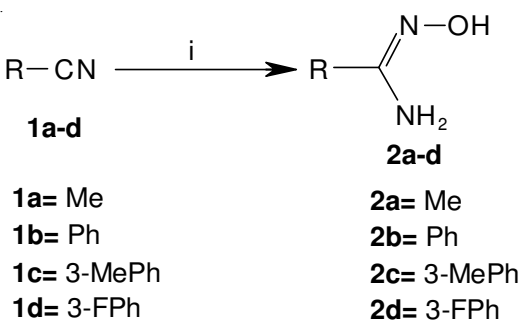

Scheme-II: Synthesis of amidoximes; Conditions: (i) Hydroxylamine. Hydrochloride, Methanol, $\mathrm{K}_{2} \mathrm{CO}_{3}$, room temperature

Various indazole carboxylic acid methyl esters 3,4,5,6 were treated with amidoximes $\mathbf{2 a - d}$ in solvents such as DMF, NMP, ethanol and toluene using different bases such as TEA, DIPEA, pyridine, $\mathrm{NaHCO}_{3}, \mathrm{~K}_{2} \mathrm{CO}_{3}, \mathrm{tBuOK}$ and $\mathrm{NaH}$ to optimize reaction conditions. We have observed that $\mathrm{K}_{2} \mathrm{CO}_{3}$ as base and toluene as solvent at $110^{\circ} \mathrm{C}$ for 6 to $12 \mathrm{~h}$ resulted in formation 3,5-disubstitued-1,2,4-oxadiazoles (3,4,5,6) (Scheme-III-VI) in high yields and good purity.

Indazole-3-carboxylic acid methyl ester (3) was prepared starting from phenyl hydrazine (8) and 4-bromobenzaldehyde (9) (Scheme-III). Phenyl hydrazine was treated with 4-bromobenzaldehyde to result 4-bromobenzalde-hydephenylhydrazone (10) in quantitative yields. Reaction of compound $\mathbf{1 0}$ with oxalylchoride provides an intermediate $\mathbf{1 1}$, which upon treatment with aluminum chloride resulted in 4-bromobenzylideneaminoisatine (12) in good yields. Hydrolysis followed by rearrangement of compound $\mathbf{1 2}$ produces the indazole-3carboxylic acid (13). Compound $\mathbf{1 3}$ was treated with dimethylsulphate in presence of potassium carbonate in acetone at reflux temperature to give desired indazole -3-carboxylic acid methyl ester (3) in good yields. The overall yields were improved by using 4-bromobenzaldehyde when compared with benzaldehyde.

The 5-(3-indazolyl)-1,2,4-oxadiazole series 3a-d (ShecmeIII) were synthesized from compound $\mathbf{3}$; compound $\mathbf{3}$ was treated with amidoximes $\mathbf{2} \mathbf{a}-\mathbf{d}$ in the presence of potassium carbonate in toluene at reflux for 6-8 $\mathrm{h}$ and resulted in compounds 3a-d in good yields.

Indazole-carboxylic acid methyl ester $(\mathbf{4}, \mathbf{5}, \mathbf{6})$ were prepared starting from respective 2-methyl-nitrobenzoic acid (14, 


\begin{tabular}{|c|c|c|c|c|c|c|c|c|c|}
\hline \multicolumn{10}{|c|}{ TABLE-1 } \\
\hline Entry & $\mathrm{R}$ & $\mathbf{R}^{1}$ & Product & Yield $(\%)^{\mathrm{a}}$ & Entry & $\mathrm{R}$ & $\mathbf{R}^{1}$ & Product & Yield $(\%)^{\mathrm{a}}$ \\
\hline 1 & $\begin{array}{l}\mathrm{CH}_{3} \\
(\mathbf{2 a})\end{array}$ & 3 & $3 \mathbf{a}$ & 80 & 11 & $\begin{array}{l}\text { 3- } \mathrm{CH}_{3} \mathrm{Ph} \\
\quad(\mathbf{2 c})\end{array}$ & 5 & $5 c$ & 70 \\
\hline 2 & $\begin{array}{l}\mathrm{Ph} \\
(\mathbf{2 b})\end{array}$ & 3 & $3 b$ & 85 & 12 & $\begin{array}{c}\text { 3-FPh } \\
\text { (2d) }\end{array}$ & 5 & $5 d$ & 60 \\
\hline 3 & $\begin{array}{c}3-\mathrm{CH}_{3} \mathrm{Ph} \\
(\mathbf{2 c})\end{array}$ & 3 & $3 c$ & 80 & 13 & $\begin{array}{l}\mathrm{CH}_{3} \\
\text { (2a) }\end{array}$ & 6 & $6 a$ & 85 \\
\hline 4 & $\begin{array}{c}\text { 3-FPh } \\
\text { (2d) }\end{array}$ & 3 & $3 d$ & 75 & 14 & $\begin{array}{l}\mathrm{Ph} \\
(\mathbf{2 b})\end{array}$ & 6 & $6 b$ & 90 \\
\hline 5 & $\begin{array}{l}\mathrm{CH}_{3} \\
(\mathbf{2 a})\end{array}$ & 4 & $4 a$ & 75 & 15 & $\begin{array}{c}3-\mathrm{CH}_{3} \mathrm{Ph} \\
\quad(\mathbf{2 c})\end{array}$ & 6 & $6 c$ & 75 \\
\hline 6 & $\begin{array}{l}\mathrm{Ph} \\
\text { (2b) }\end{array}$ & 4 & $4 b$ & 85 & 16 & $\begin{array}{c}\text { 3-FPh } \\
\text { (2d) }\end{array}$ & 6 & $6 d$ & 72 \\
\hline 7 & $\begin{array}{c}3-\mathrm{CH}_{3} \mathrm{Ph} \\
(\mathbf{2 c})\end{array}$ & 4 & $4 c$ & 80 & 17 & $\begin{array}{l}\mathrm{CH}_{3} \\
\text { (2a) }\end{array}$ & 24 & $27 a$ & 72 \\
\hline 8 & $\begin{array}{c}\text { 3-FPh } \\
\text { (2d) }\end{array}$ & 4 & $4 d$ & 70 & 18 & $\begin{array}{l}\mathrm{Ph} \\
(\mathbf{2 b})\end{array}$ & 24 & $27 b$ & 79 \\
\hline 9 & $\begin{array}{l}\mathrm{CH}_{3} \\
(\mathbf{2 a})\end{array}$ & 5 & $5 a$ & 65 & 19 & $\begin{array}{l}\text { 3- } \mathrm{CH}_{3} \mathrm{Ph} \\
\quad(\mathbf{2 c})\end{array}$ & 24 & $27 c$ & 80 \\
\hline 10 & $\begin{array}{l}\mathrm{Ph} \\
(\mathbf{2 b})\end{array}$ & 5 & $5 b$ & 75 & 20 & $\begin{array}{c}\text { 3-FPh } \\
\text { (2d) }\end{array}$ & 24 & 27d & 71 \\
\hline
\end{tabular}

${ }^{a}$ Purified products.

17, 20) (Scheme-IV); compound 14, 17, 20 were converted to methyl ester $(\mathbf{1 5}, \mathbf{1 8}, \mathbf{2 1})$ with dimethylsulphate in presence of potassium carbonate in acetone at reflux temperature. The compound nitro ester 15, 18, 21 were treated with $\mathrm{Fe} / \mathrm{NH}_{4} \mathrm{Cl}$ at reflux temperature to affordcorresponding 16, 19, 22 in good yields. Products 16, 19, 22 were treated with sodium nitrite to get hydrazine followed by cyclization results in indazolecarboxylic acid methyl ester $(\mathbf{4}, \mathbf{5}, \mathbf{6})$ (Scheme-IV).
5-Indazolyl-1,2,4-oxadiazole 4a-d, 5a-d, 6a-d (SchemeIV) were synthesized from compound 4, 5, 6; compound 4, 5, $\mathbf{6}$ were treated with amidoximes $\mathbf{2 a - d}$ in the presence of potassium carbonate in toluene resulted in good yields.

Synthesis of 7-indazolyl-1,2-4-oxadiazoles were unsuccessful to get the desired product when we adopted regular procedure to make the indazoles followed by oxadiazoles. 2-Methyl-3-nitro-benzoic acid (23) was converted to methyl 


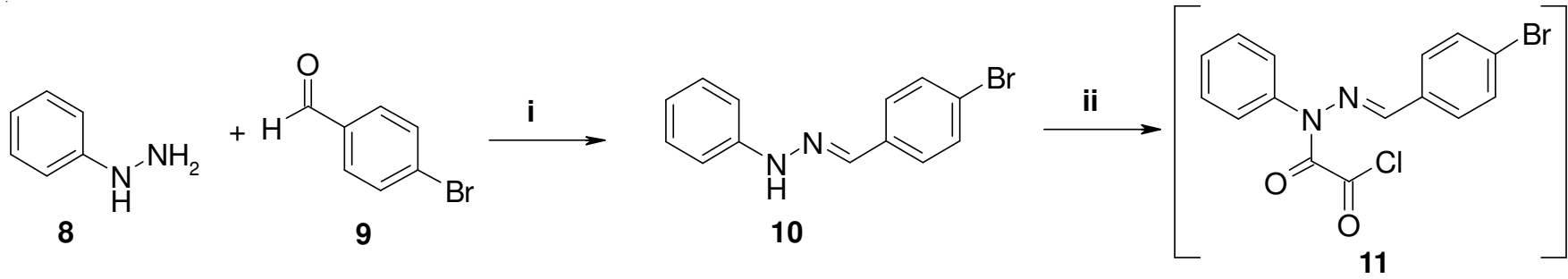

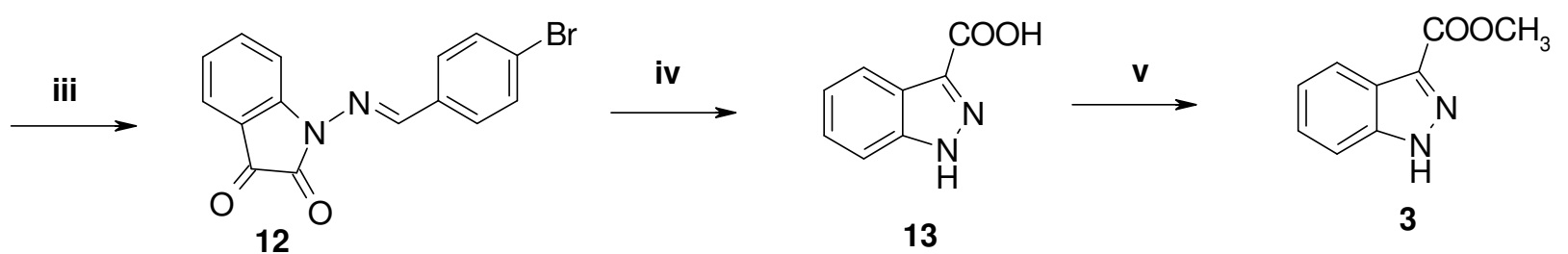<smiles>[R]c1noc(-c2n[nH]c3ccccc23)n1</smiles>

Scheme-III: Synthesis of 5-(3-indazolyl)-1,2,4-oxadiazole; Conditions: (i) Water, 25 to $35{ }^{\circ} \mathrm{C}, 95 \%$; (ii) Oxalyl chloride, DCM, reflux; (iii) AlCl 3 , DCM, reflux, $91 \%$ for two steps; (iv) Conc. $\mathrm{HCl}, \mathrm{AcOH}, 95^{\circ} \mathrm{C}, 85 \%$; (v) $\mathrm{K}_{2} \mathrm{CO}_{3}$, DMS, Acetone, reflux, $95 \%$; (vi) Amidoxime $(\mathbf{2 a - d} ; \mathbf{a}=\mathrm{Me}, \mathbf{b}=$ $\mathrm{Ph}, \mathbf{c}=3-\mathrm{MePh}, \mathbf{d}=3-\mathrm{FPh}), \mathrm{K}_{2} \mathrm{CO}_{3}$, Toluene, reflux<smiles>Cc1ccc(C(=O)O)cc1[N+](=O)[O-]</smiles>

$14,17,20$
$15,18,21$<smiles>C#CCC#CC</smiles>

$16,19,22$<smiles></smiles>

$4,5,6$

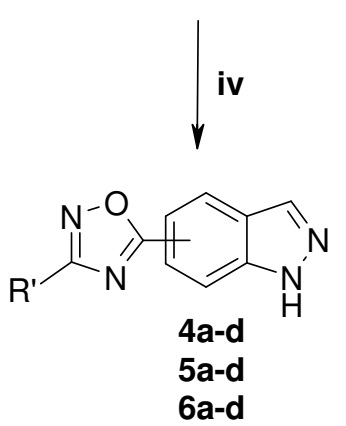

Scheme-IV: Synthesis of 5-indazolyl-1,24-oxadiazole; Conditions: (i) $\mathrm{K}_{2} \mathrm{CO}_{3}$, DMS, Acetone, reflux; (ii) $\mathrm{Fe}, \mathrm{NH}_{4} \mathrm{Cl}$, $\mathrm{EtOH}$, $\mathrm{H}_{2} \mathrm{O}, 65$ to $75{ }^{\circ} \mathrm{C}$; (iii) Isoamylnitrite, $\mathrm{Ac}_{2} \mathrm{O}, \mathrm{KOAc}, \mathrm{CHCl}_{3}$, Reflux or $\mathrm{NaNO}_{2}, \mathrm{AcOH}, \mathrm{H}_{2} \mathrm{O}$, rt; (iv) Amidoxime (2a-d, a = Me, b = Ph, c = 3-MePh, 4-3- $\mathrm{FPh}$ ), $\mathrm{K}_{2} \mathrm{CO}_{3}$, toluene, $110^{\circ} \mathrm{C}$; (iii)

ester (24) with dimethyl sulphate in presence of potassium carbonate in acetone at reflux temperature (Scheme-V). Compound 24 was treated with $\mathrm{Fe} / \mathrm{NH}_{4} \mathrm{Cl}$ at reflux temperate to get compound $\mathbf{2 5}$ in good yields. Compound $\mathbf{2 5}$ was treated with sodium nitrite to get corresponding hydrazine followed by cyclization to get indazole-7-carboxylic acid methyl ester (7) was unsuccessful (Scheme-V). The hydrazine found at step-3 reacted with the adjacent ester group to from cyclic hydrazone (26). Hence a different approach was attempted to make these analogues as discussed below.

In the modified approach construction of the oxadiazole ring was carried out first and later we carried out indazole ring formation. Compound $\mathbf{2 4}$ was treated with amidoxime (2a-d) in presence of potassium carbonate in toluene at reflux temperature to provide oxadiazole (27a-d). Nitro group of compound 27a-d was reduced with iron and ammonium chloride in ethanol at reflux temperature to produce corresponding amines 28a-d, which on reaction with isoamylnitrite, $\mathrm{Ac}_{2} \mathrm{O}$ and potassium acetate in chloroform at reflux gave the desired 5-(7indazolyl)-1,2-4-oxadiazoles (7a-d) (Scheme-VI, Table-2).

As an alternative, we have also studied the synthesis of 3,5-disubstitued-1,2,4-oxazidazoles 3,4,5,6,7 (Scheme-VII), using amidoximes 2a-d and carboxylic acid $\mathbf{1 3}$ in presence of coupling agents such as DCC, EDC and HOBt and found that 
<smiles>Cc1cccc(C(=O)O)c1[N+](=O)[O-]</smiles>

23<smiles>COC(=O)c1cccc(C)c1[N+](=O)[O-]</smiles>

24<smiles>C=CC</smiles><smiles>CC=C(CC)C(=O)OC</smiles>

25<smiles>Cc1cccc2c1N=NC2=O</smiles>

Scheme-V: $\quad$ Synthesis of methyl-7-indazole carboxylic acid (7); Conditions: (i) DMS, $\mathrm{K}_{2} \mathrm{CO}_{3}$, Acetone, Reflux, $90 \%$; (ii) $\mathrm{Fe}, \mathrm{NH}_{4} \mathrm{Cl}, \mathrm{EtOH}_{2} \mathrm{H}_{2} \mathrm{O}, 65$ to $75^{\circ} \mathrm{C}, 85 \%$; (iii) Isoamylnitrite, $\mathrm{Ac}_{2} \mathrm{O}, \mathrm{KOAc}, \mathrm{CHCl}_{3}$, Reflux or $\mathrm{NaNO}_{2}, \mathrm{AcOH}, \mathrm{H}_{2} \mathrm{O}$, rt<smiles>COC(=O)c1cccc(C)c1[N+](=O)[O-]</smiles>

24<smiles>[R]c1noc(-c2cccc(C)c2[N+](=O)[O-])n1</smiles>

27a-d

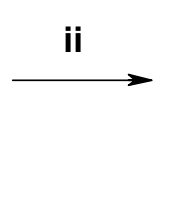<smiles>[R]c1noc(-c2cccc3cn[nH]c23)n1</smiles>

7a-d

Scheme-VI: Synthesis of 5-(7-indazolyl)-1,2-4-oxadiazoles; Conditions: (i) Amidoxime (2a-d, $\mathbf{a}=\mathbf{M e}, \mathbf{b}=\mathbf{P h}, \mathbf{c}=\mathbf{3}-\mathbf{M e P h}, \mathbf{d}=\mathbf{3}-\mathbf{F P h}$ ), $\mathrm{K}_{2} \mathrm{CO}, \mathrm{Toluene}$,

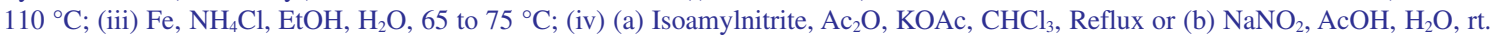

\begin{tabular}{cccc}
\multicolumn{4}{c}{ TABLE-2 } \\
\hline Entry & $\mathrm{R}$ & Product & Yield $(\%)^{\mathrm{a}}$ \\
\hline 1 & $\mathrm{CH}_{3}$ & $\mathbf{7 a}$ & 78 \\
2 & $\mathrm{Ph}$ & $\mathbf{7 b}$ & 81 \\
3 & $3-\mathrm{MePh}$ & $\mathbf{7 c}$ & 76 \\
4 & $3-\mathrm{FPh}$ & $\mathbf{7 d}$ & 72 \\
\hline
\end{tabular}

${ }^{a}$ Purified products<smiles>[R]C(N)=NO</smiles>

2a: $R=M e$

2b: $R=P h$

2c: $\mathrm{R}=3-\mathrm{CH}_{3} \mathrm{Ph}$

2d: $R=3-F P h$

13<smiles>[R]c1noc(-c2n[nH]c3ccccc23)n1</smiles>

3a-d

3a: $\mathrm{R}=\mathrm{Me}$

3b: $\mathbf{R}=\mathbf{P h}$

3c: $\mathbf{R}=3-\mathrm{CH}_{3} \mathrm{Ph}$

3d: $\mathbf{R}=3-\mathrm{FPh}$
Scheme-VII: Synthesis of 3-indazolyl-1,2-4-oxadiazoles; Conditions: (i) (a) DCC, HOBt, DCM, rt; (b) Pyridine, reflux.

the yields of oxadiazoles were much lower when compared with $\mathrm{K}_{2} \mathrm{CO}_{3}$ toluene procedure from corresponding esters.

All new compounds $\mathbf{3}, \mathbf{4 , 5}, \mathbf{6}, \mathbf{7}$ were thoroughly characterized by ${ }^{1} \mathrm{H}$ and ${ }^{13} \mathrm{C}$ NMR, Mass and IR spectra.

\section{Conclusion}

The work presented here demonstrates a straight forward and mild procedure for an efficient synthesis of 3,5-disubstituted-1,2,4-oxadiazoles using potassium carbonate as base and toluene as solvent. A variety of esters can be used to expand the scope of substituents around the oxadiazole ring. We have also successfully demonstrated the synthesis of all the positional isomers of indazolyl (3-7 positions) oxadiazoles in good yields. The high pharmacological importance of indazoles and oxadiazoles prompted us to synthesize these derivatives.

\section{ACKNOWLEDGEMENTS}

The authors thank to Dr. C. Satyanarayana, CEO, Laurus Labs, for his cooperation and encouragement.

\section{REFERENCES}

1. (a) G.D. Diana, D.L. Volkots, T.J. Nitz, T.R. Baily, M.A. Long, N. Vescio, S. Aldous, D.C. Pevear and F.J. Dutko, J. Med. Chem., 37, 2421 (1994); (b) S. Borg, R.C. Vollinga, M. Labarre, K. Payza, L. Terenius and K. Luthman, J. Med. Chem., 42, 4331 (1999).

2. H.Z. Zhang, S. Kasibhatla, J. Kuemmerle, W. Kemnitzer, K. OllisMason, L. Qiu, C. Crogan-Grundy, B. Tseng, J. Drewe and S.X. Cai, J. Med. Chem., 48, 5215 (2005).

3. Z. Li, W. Chen, J.J. Hale, C.L. Lynch, S.G. Mills, R. Hajdu, C.A. Keohane, M.J. Rosenbach, J.A. Milligan, G.J. Shei, G. Chrebet, S.A. Parent, J. Bergstrom, D. Card, M. Forrest, E.J. Quackenbush, L.A. Wickham, H. Vargas, R.M. Evans, H. Rosen and S. Mandala, J. Med. Chem., 48, 6169 (2005). 
4. J. Roppe, N.D. Smith, D. Huang, L. Tehrani, B. Wang, J. Anderson, J. Brodkin, J. Chung, X. Jiang, C. King, B. Munoz, M.A. Varney, P. Prasit and N.D.P. Cosford, J. Med. Chem., 47, 4645 (2004).

5. L.J. Street, R. Baker, T. Book, C.O. Kneen, A.M. MacLeod, K.J. Merchant, G.A. Showell, J. Saunders and R.H. Herbert, J. Med. Chem., 33, 2690 (1990)

6. C.J. Swain, R. Baker, C. Kneen, J. Moseley, J. Saunders, E.M. Seward, G. Stevenson, M. Beer, J. Stanton and K. Watling, J. Med. Chem., 34, 140 (1991).

7. F. Watjen, R. Baker, M. Engelstoff, R. Herbert, A. Macleod, A. Knight, K. Merchant, J. Moseley and J. Saunders, J. Med. Chem., 32, 2282 (1989).

8. F.I. Carroll, J.L. Gray, P. Abraham, M.A. Kuzemko, A.H. Lewin, J.W. Boja and M.J. Kuhar, J. Med. Chem., 36, 2886 (1993).

9. S. Borg, G. Estenne-Bouhtou, K. Luthman, I. Csoeregh, W. Hesselink and U. Hacksell, J. Org. Chem., 60, 3112 (1995).

10. J.L. Buchanan, C.B. Vu, T.J. Merry, E.G. Corpuz, S.G. Pradeepan, U.N Mani, M. Yang, H.R. Plake, V.M. Varkhedkar, B.A. Lynch, I.A. MacNeil, K.A. Loiacono, C.L. Tiong and D.A. Holt, Bioorg. Med. Chem. Lett., 9, 2359 (1999).

11. P.L. Durette, W.K. Hagmann, I.E. Kopka and M. MacCoss, WO 00/ 71572 A1 (2000).

12. D.M. Cottrell, J. Capers, M.M. Salem, K. DeLuca-Fradley, S.L. Croft and K.A. Werbovetz, Bioorg. Med. Chem., 12, 2815 (2004).

13. D.D. Feng, T. Biftu, M.R. Candelore, M.A. Cascieri, L.F. Colwell Jr., L. Deng, W.P. Feeney, M.J. Forrest, G.J. Hom, D.E. MacIntyre, R.R. Miller, R.A. Stearns, C.D. Strader, L. Tota, M.J. Wyvratt, M.H. Fisher and A.E. Weber, Bioorg. Med. Chem. Lett., 10, 1427 (2000).

14. D.N. Nicolaides, K.C. Fylaktakidou, K.E. Litinas and D. HadjipavlouLitina, Eur. J. Med. Chem., 33, 715 (1998).

15. S. Cesarini, N. Colombo, M. Pulici, E.R. Felder and W. Brill, Tetrahedron, 62, 10223 (2006).

16. C.O. Kangani, D.E. Kelley and B. Day, Tetrahedron Lett., 47, 6497 (2006).
17. V. Polshettiwar and R.S. Varma, Tetrahedron Lett., 49, 879 (2008).

18. H.A. Rajapakse, H. Zhu, M.B. Young and B.T. Mott, Tetrahedron Lett., 47, 4827 (2006).

19. G.N. Vazquez, G.M. Molina-Salinas, Z.V. Duarte-Fajardo, J.V. Villarreal, S.E. Soto, F.G. Salazar, E.H. Nunes and S.S. Fernandez, Bioorg. Med. Chem., 15, 5502 (2007).

20. M. Ankersen, B. Peschke, B.S. Hansen and T.K. Hansen, Bioorg. Med. Chem. Lett., 7, 1293 (1997).

21. C.B. Vu, E.G. Corpuz, T.J. Merry, S.G. Pradeepan, C. Bartlett, R.S. Bohacek, M.C. Botfield, C.J. Eyermann, B.A. Lynch, I.A. MacNeil, M.K. Ram, M.R. van Schravendijk, S. Violette and T.K. Sawyer, J. Med. Chem., 42, 4088 (1999).

22. J. Matsumoto, T. Takahashi, M. Agata, H. Toyofuku and N. Sasada, Jpn. J. Pharmacol., 65, 51 (1994).

23. K. Ohmoto, T. Yamamoto, T. Horiuchi, H. Imanishi, Y. Odagaki, K. Kawabata, T. Sekioka, Y. Hirota, S. Matsuoka, H. Nakai, M. Toda, J.C. Cheronis, L.W. Spruce, A. Gyorkos and M. Wieczorek, J. Med. Chem., 43, 4927 (2000).

24. J. Rudolph, H. Theis, R. Hanke, R. Endermann, L. Johannsen and F.U. Geschke, J. Med. Chem., 44, 619 (2001).

25. S. Chiou and H.J. Shine, J. Heterocycl. Chem., 26, 125 (1989).

26. A.R. Gangloff, J. Litvak, E.J. Shelton, D. Sperandio, V.R. Wang and K.D. Rice, Tetrahedron Lett., 42, 1441 (2001).

27. (a) T.L. Deegan, T.J. Nitz, D. Cebzanov, D.E. Pufko and J.A. Porco Jr., Bioorg. Med. Chem. Lett., 9, 209 (1999); (b) R.F. Poulain, A.L. Tartar and B.P. Deprez, Tetrahedron Lett., 42, 1495 (2001).

28. C.D. Bedford, R.A. Howd, O.D. Dailey, A. Miller, H.W. Nolen, R.A. Kenley, J.R. Kern and J.S. Winterle, J. Med. Chem., 29, 2174 (1986).

29. (a) K. Rice and J.M. Nuss, Bioorg. Med. Chem. Lett., 11, 753 (2001); (b) A.R. Gangloff, J. Litvak, E.J. Shelton, D. Sperandio, V.R. Wang and K.D. Rice, Tetrahedron Lett., 42, 1441 (2001).

30. (a) Y. Wang, R.L. Miller, D.R. Sauer and S.W. Djuric, Org. Lett., 7, 925 (2005); (b) M. Adib, A.H. Jahromi, N. Tavoosi, M. Mahdavi and H.R. Bijanzadeh, Tetrahedron Lett., 47, 2965 (2006). 